\title{
Bibliometrics EEG metrics associations and connections between military medicine and the differentiate post traumatic stress disorder (PTSD)
}

\author{
VASILEIOS STEFANIDIS ${ }^{1}$, SOZON PAPAVLASOPOULOS ${ }^{1}$, MARIOS POULOS ${ }^{1}$, \\ NIKOS BARDIS ${ }^{2}$ \\ ${ }^{1}$ Information Science and Informatics Ionian University Corfu, Corfu, GREECE \\ ${ }^{2}$ Hellenic Military Academy, Vari, GREECE
}

\begin{abstract}
In this paper we investigate the bibliometric association and connection between Electroencephalography (EEG) metrics of human brain and connections between military medicine and the post traumatic stress disorder (PTSD). In EEG metrics included various metrics used from scientists in order to map the brain activity. Inrecent years there has been an increasingly amount of data connect EEG metrics with PTSD and military medicine. Due to breakthroughs in biology and bioinformatics, more and more data are stored in various large databases as biomedical databases. In recent years, biomedical information has become the center of research, and its data volume has continued to grow. Therefore, obtaining effective information from scientists has become increasingly challenging. As a new scientific field of bioinformatics, new tools and applications are needed to extract important scientific data based on experimental results and information provided by papers and journals. In this paper we are going to investigate methods based in acustom made IT system, more specifically a programmable in Python toolin order to find connections between the differentiate post traumatic stress disorder and the brain operation and signaling. This IT system could become a useful tool against the struggle of scientists and medicalprofessionalsin the near future.
\end{abstract}

Key-Words: - EEG metrics, MedLine, Bioinformatics, Co-citation coupling, Bibliometrics, PubMed, Power Spectra, PTSD, ERPs, EEG Entropy, EEG Rhythms

Received: March 30, 2021. Revised: April 28, 2021. Accepted: May 10, 2021. Published: May 19, 2021.

\section{Introduction}

The study of various Electroencephalography (EEG) metrics is a biometric method that can give us fairly detailed information on ongoing brain activity associated with perception, cognition and emotion [1],[2], [6], [19].

Post-traumatic stress disorder (PTSD) is a common psychiatric condition amongst active duty and ex-serving military personnel across the world, creating a significant public health challenge [[20]]. In [[25]] researchers investigate the use of restingstate electroencephalography (EEG) data to help differentiate posttraumatic stress disorder (PTSD) symptom factors. In this research there is a correlation between PTSD and EEG Rhythms (alpha, beta, delta, and theta frequency band).

In [[26]] researchers examine electroencephalogram (EEG) spectral power to study abnormalities in regional brain activity in posttraumatic stress disorder (PTSD) during sleep. According to auhtors they aimed to identify sleep EEG markers of PTSD that were reproducible across nights and subsamples of our study population.
There are various EEG-based Metrics $[[6]],[[23]],[[24]]$ we can find in literature as cognitive state for engagement and distraction as well as a mental workload metric [17], the memory workload, sort memory capacity, various EEG entropies, power spectra (PS), coherence (ITC InterTrial Coherence) [19], the pre-stimulus noise (PSN), signal-to-noise ratio (SNR) and EEG amplitude variance across the P300 event window (CVERP) [18], the EEG rhythms, event related potentials (ERPs) [20] etc. Some of them are connected in literature with military medicine and the differentiate post traumatic stress disorder (PTSD). Generally those metrics enable researchers to observe the unobservable brain, and incorporate a useful and reliable alternative to analyzing raw EEG data.

The study of the entropy of a signal is a study based on the theory of chaos. Techniques of this type appear to be better able to deal with stochastic systems. If a stochastic system has an entropy of zero then it is random and any increase in entropy increases randomness. Stochastic is a system that is not deterministic. A system that is described by the theory of probabilities. Entropy from the point of 
view of Physics is considered to be a magnitude which expresses the measure of the disorder of a system.

Bioinformatics is a scientific field whose main purpose is to analyze statistical data and classify the flow of information generated by scientific experiments or laboratory work. Scientists can use various methods to analyze scientific data and evaluate huge data sets. In the past few decades, these tasks have been very difficult. On the one hand, due to limited computing power and lack of interdisciplinary fields like bioinformatics, it can be used. In the past few years, as mentioned above, a large amount of data has begun to accumulate. The main question is how to use these massive amounts of data in the future. Therefore, new technologies must be found. Several attempts have been made to extract information from various scientific papers and/or journals freely available on the Internet. We can find various techniques in this way, in the previous years as at Stapley et al [3]. In this paper the researchers have introduced term "biobibliometrics" to describe the use of bibliometric techniques on papers that are related to biological issues. We can apply the technique in a similar way for educational disorders issues. The specific implementation we can find at Stapley's could verify the bibliometrical connection between biological data as the EEG metrics, based solely on the rate of their common appearances in the abstracts of scientific papers and journals.

In this paper, we are going to investigate various methods and finally to use a custom made IT systemin order to find connections between Posttraumatic stress disorder (PTSD) and the brain operation and signaling. EEG is the main system for register the brain activity. Thus EEG is a significant medical issue. As first step we use a previous implemented IT system [[23]] [[24]] in order to search in the free full-text archive of Educational disorders, biomedical and life sciences journal literature at the U.S. in order to investigate the possible association between the selected disorders and the various EEG metrics.

The present paper is divided in the following three parts: The "methodology" in which is explained the methodology we develop. The second part concerns the "results" and the third part includes some discussion and the final conclusion

\section{Experimental and Computational Details}

The EEG metrics that will be studied in this work in order to draw and possible correlation with PTSD are Power Spectra (PS), ERPs, EEG Rhythms and EEG entropies. The primary and basic goal of our IT system will be the ability to search for those EEG metrics supplied by the user and identify any connections or interactions with Post-traumatic stress disorder based on how frequently they are met together in several papers stored in PubMed (US National Library of Medicine National Institutes of Health) central database [5].

\subsection{Methodology}

Based on the principals of bibliometrics and statistics the system will take into account a series of parameters in order to create a weight-graph between metrics and learning disorders. The basic parameters will be:

- Frequency of the co-appearance of two EEG metrics in the abstracts of papers, freely available online with no restrictions

- EEG metrics and PTSD Co-citation coupling [15]

- Analysis of related EEG metricsand PTSDdata in pairs

- Analysis of the probability of relation between EEG metrics and PTSDdata that coexist in several papers based on the Pubmed Central Database

\subsection{High Level Design}

Our IT system constantly poll for EEG metrics and analyze their appearance in papers stored in PubMed[5]. It will then store and link this information when it is requested by the user. For example when EEG PS is analyzed the system will store the PID (Paper ID) of PubMed for each paper that contains EEG PS (metric 1). Then the same procedure is going to be followed for ERPS (metric 2), EEG Rhythms (metric 3) and EEG entropies (metric 4) and finally with PTSD (metric 5). The system based on the user input will construct relations between metrics following the basic principles mentioned above. This procedure will be running in real time and will update the information of each metric since the amount of papers being submitted every day could change the final graphs dramatically. 
Table 1.

\begin{tabular}{|c|c|c|c|c|c|c|c|c|c|c|c|}
\hline & PTSD & $\begin{array}{c}\text { EEG } \\
\text { Entropy }\end{array}$ & PS & ERPs & $\begin{array}{c}\text { EEG } \\
\text { Rhythms }\end{array}$ & & PTSD & $\begin{array}{c}\text { EEG } \\
\text { Entropy }\end{array}$ & PS & ERPS & $\begin{array}{c}\text { EEG } \\
\text { Rhythms }\end{array}$ \\
\hline \multicolumn{12}{|l|}{ PTSD } \\
\hline $\begin{array}{l}5890 \text { papers } \\
\text { PS }\end{array}$ & $15,55 \%$ & & $21,19 \%$ & $31,51 \%$ & $27,03 \%$ & $\begin{array}{l}\text { EEG Entropy } \\
5890 \text { papers }\end{array}$ & 916 & & 1248 & 1856 & 1592 \\
\hline $\begin{array}{c}10405 \text { papers } \\
\text { ERPs }\end{array}$ & $4,21 \%$ & $11,99 \%$ & & $48,55 \%$ & $46,09 \%$ & $\begin{array}{c}\text { PS } \\
10405 \text { papers }\end{array}$ & 438 & 1248 & & 5052 & 4796 \\
\hline 17875 papers & $5,12 \%$ & $8,91 \%$ & $26,83 \%$ & $42,49 \%$ & & EEG Rhythms & 916 & 1592 & 4796 & 7595 & \\
\hline
\end{tabular}

Constructing relations between metrics

\begin{tabular}{cc}
\hline Metric ID & Metric ID \\
\hline Metric_i & Metric_j \\
\hline PID & PID \\
\hline $0001 \longrightarrow 001$ \\
0003 & 0002 \\
$0005 \longrightarrow 0005$ \\
$0006 \longrightarrow 006$ \\
0010 & 0011 \\
\hline
\end{tabular}

As seen on Table1 we could construct a relation node between metric 1 and metric 2 with the weight of 3.

\section{Experimental Results}

We are going to use the findings of our previous study [23],[24] to identify possible bibliographic relationship between the various EEG metrics, as EEG Entropy, Power Spectra (PS) and Posttraumatic stress disorder (PTSD). In order to do so, we have applied a searching mechanism based, on an IT system programmed in python, via PCM of PubMed services and the results are presented in Table 2 and Table 3.

Table 2. Co-appearances between metrics (papers)
Table 3. Co-appearances between metrics (per cent)

\subsection{Investigating the relations between metrics}

The purpose of this subsection is the investigation of possible connections between the PTSD and ERPs and EEG Rythms metrics. For this reason we correlate the possible connection between the mentioned metrics. By analyzing the data from Table 1 and analyzing the connections between the above mentioned metrics we obtained the following results which are depicted in Figure 1.

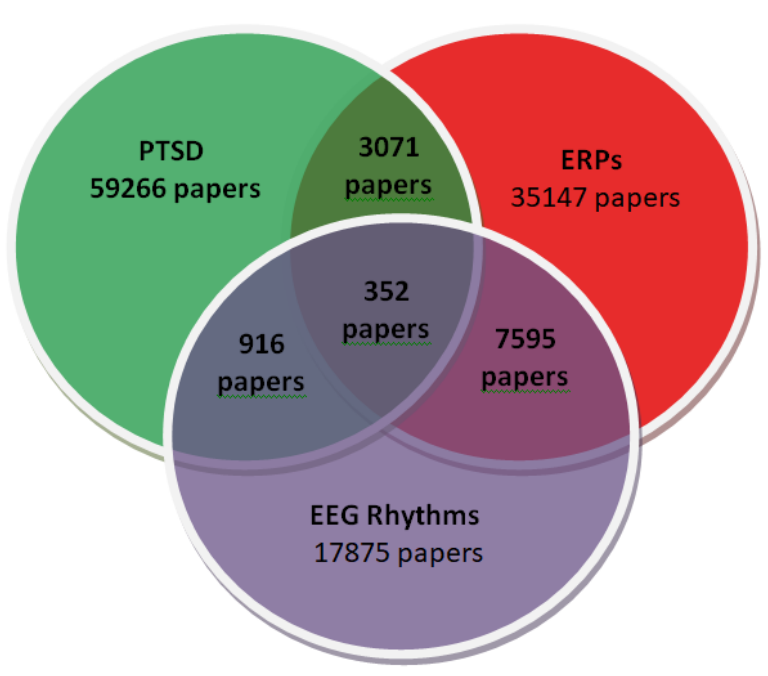

Fig. 1. The intersection between PTSD and ERPs with EEG Rhythms

According to the above results now it is possible to apply the co-citation normalization procedure [16] which is based on the following equation

$$
\text { norm }=\frac{\mid \text { in }(P T S D) \cap i n(E R P) \cap i n(E E G R y t h m s) \mid}{i n(P T S D) \cap i n(E R P)}=\frac{352}{3071}=0.11
$$


norm $=\frac{\mid \text { in }(\text { PTSD }) \cap i n(E R P) \cap \text { in }(\text { EEGRythms }) \mid}{\operatorname{in}(P T S D) \cap i n(\text { EEGRythms })}=\frac{352}{916}=0.38$

The interpretation of this result indicates that the value 0.11 from equation (1) gives a possible bringing between PTSD and ERPs metrics at $11 \%$ and the value 0.38 from equation (2) gives a possible bringing between PTSD and EEG Rythms metrics at $38 \%$. This lead the ascertainment that a possible research in this issue obtains a higher successful rate than previous attempts.

\subsection{Observing the relation between EEG metrics over time}

The purpose of this subsection is the visualization of the relations we reveal in the previous subsection. Also we can draw conclusions by observing this visualization doing. In order to visualize the relation between PTSD and EEG metrics, Power Spectra, ERPs, EEG Entropies and Rhythms we are going to investigate the appearance of each metric, in PubMed Central Database, over the past 16 months with one month interval (Figure 2). We have also determined the relation between PTSD and EEG Rhythms, and between PTSD and ERPs over the period of 16 months, again with one month interval (Figure 3). All the data used to construct the graphs in figure 2 and 3 can be observed in detail in Table 3 .

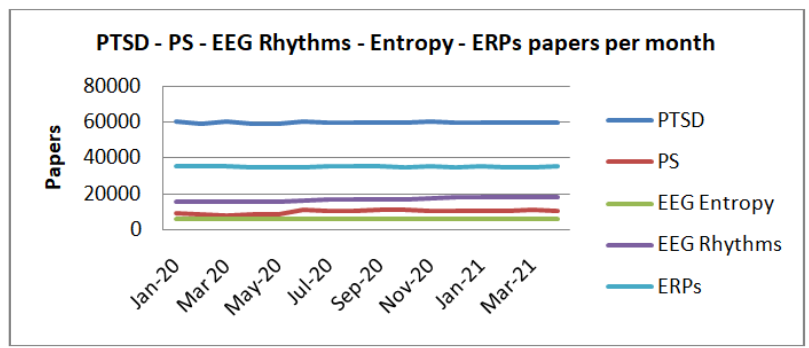

Fig. 2 Appearances of Power Spectra, EEG Rhythms and ERPs in PubMed Central over the past 16 months.

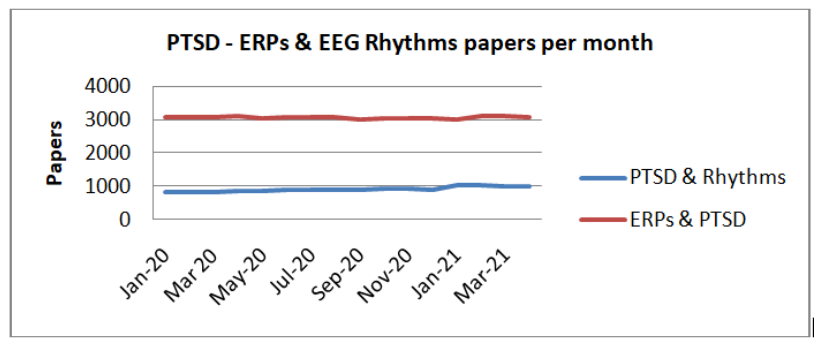

Fig. 3 Number of co-appearances between EEG Rhythms and Power Spectra and between EEG Rhythms and ERPs in PubMed Central over the past 16 months.

Table 4. Appearance and co-appearances of PTSD, Power Spectra (PS), EEG Entropies, EEG Rhythms and ERPs

\begin{tabular}{lccccccc}
\hline & PTSD & PS & $\begin{array}{c}\text { EEG } \\
\text { Entropy }\end{array}$ & $\begin{array}{c}\text { EEG } \\
\text { Rhythms }\end{array}$ & ERPs & $\begin{array}{c}\text { PTSD \& } \\
\text { Rhythms }\end{array}$ & $\begin{array}{c}\text { ERPs \& } \\
\text { PTSD }\end{array}$ \\
\hline Jan-20 & 59821 & 8970 & 5919 & 15786 & 35234 & 820 & 3081 \\
Feb-20 & 59265 & 8708 & 5761 & 15839 & 35343 & 831 & 3072 \\
Mar 20 & 59845 & 8044 & 6056 & 15828 & 35372 & 814 & 3066 \\
Apr-20 & 59241 & 8908 & 5859 & 15908 & 35121 & 849 & 3096 \\
May-20 & 59316 & 8693 & 5881 & 15959 & 35010 & 840 & 3030 \\
Jun-20 & 59865 & 10686 & 5887 & 16226 & 35218 & 885 & 3058 \\
Jul-20 & 59668 & 10396 & 5725 & 16720 & 35308 & 899 & 3052 \\
Aug-20 & 59438 & 10203 & 6080 & 16743 & 35446 & 876 & 3077 \\
Sep-20 & 59709 & 10748 & 5769 & 16852 & 35249 & 888 & 3005 \\
Oct-20 & 59713 & 10941 & 5850 & 17100 & 35168 & 932 & 3021 \\
Nov-20 & 59945 & 10302 & 5841 & 17456 & 35333 & 933 & 3035 \\
Dec-20 & 59537 & 10313 & 6063 & 17925 & 35181 & 900 & 3029 \\
Jan-21 & 59734 & 10411 & 5765 & 17897 & 35457 & 1045 & 3000 \\
Feb-21 & 59673 & 10117 & 5865 & 17994 & 35048 & 1043 & 3087 \\
Mar-21 & 59654 & 10849 & 5862 & 17811 & 35147 & 1005 & 3100 \\
Apr-21 & 59621 & 10132 & 5715 & 17762 & 35425 & 1005 & 3060
\end{tabular}

As we can observe in Figures 2 and 3 there is a noticeable increase in EEG rhythms appearances in journals, Power Spectra appearance rates seem to be stable and there is a slight increase in the appearances of ERPs. We can also notice that there is a slight increase of the bio-bibliometric relation between PTSD and EEG Rhythms. Finally we identify an almost stable relation between PTSD and ERPs.

\section{Results and Discussion}

The bibliometric approach we presented in this paper, between Post-traumatic stress disorder (PTSD) and Human brain via EEG metrics, could provide a very important tool for the scientific community, improving connections between learning disabilities and human brain EEG metrics. In this paper we showed that the EEG Rhythms metric plays a crucial role regarding the identification of possible relation between EEG and PTSD. Through this paper we showed that if we study the graphs provided we are sure that EEG Rhythms could be the common link between EEG metrics affecting possibility of learning disability existence. Thus we believe that further pursue of this work could be made by taking into account the crucial role of the EEG Rhythms metric.

We have the opinion that the statistical system will become a useful tool for researchers around the world. The specific implementation will provide a 
mean to connect, seemingly unconnected PTSD and EEG metrics.

\section{Conclusions and Future Work}

In this paper we approached with bibliometric terms the scientific fields of military medicine and Electroencephalography (EEG) metrics. More specifically we presented association and communication between post-traumatic stress disorder (PTSD) and human brain through various EEG metrics. We used EEG Entropies, Power Spectra, Event Related Potentials, EEG Rhythms Oscillations. As a conclusion we can conclude with that, via this paper we provide with a very important tool the scientific community, improving connections between post-traumatic stress disorder (PTSD) and human brain EEG metrics. In this paper we showed that the EEG Rhythms metric plays a crucial role regarding the identification of possible relation between EEG and PTSD. Based on the graphs provided we are fairly sure that EEG Rhythms could be the guide and the common link between EEG metrics affecting possibility of learning disability existence. For this reason we believe that further pursue of this work could be made by taking into account the crucial role of the EEG Rhythms metric. We have the opinion that the statistical system will become a useful tool for researchers around the world. The specific implementation will provide a mean to connect, seemingly unconnected PTSD and EEG metrics.

As future work, we plan to develop a parametric information system in order to automate the statistical procedure of tables 2,3 and 4 . The information system will constitute from an intelligent calculate mechanism based on Python and a graphical user interface with parameters that can automatically extract bibliometric information and statistical information from PubMed or other online databases.

\section{References:}

[1] Michael L. Metzker ," Sequencing technologies - the next generation" Nature Reviews Genetics 11, 31-46 ,January 2010.

[2] Jacques Cohen, "Bioinformatics - an introduction for computer scientists", ACM Compu-ting Surveys, Volume 36 Issue 2,122-158,June 2004

[3] B.J. Stapley et al,"Biobibliometrics: Information retrieval and visualization from co-occurrences of genenames in Medline abstracts", Pacific Symposium on Biocomputing 5:526-537 (2000)
[4] Martzoukos Y., Papavlasopoulos S., Poulos M., Syrrou M., "Biobibliometrics \& gene con-nections",IISA2015

[5] http://www.ncbi.nlm.nih.gov/pme/ $(10 / 04 / 2015)$

[6] Poulos M. and Stefanidis V. , "Synchronization of Small Set Data on Stable Period", 2nd International Conference Mathematics and Computers in Science and Industry, Malta, 2015.

[7] Baars, B. J., Gage, N. M., (2010), Cognition, Brain, and Consciousness: Introduction to Cognitive Neuroscience, Elsevier, 2nd Edition.

[8] Hodgkinson, Colin A., Mary-Anne Enoch, Vibhuti Srivastava, Justine S. CumminsOman, Cherisse Ferrier, Polina Iarikova, Sriram Sankararaman, et al. "Genome-Wide Associa-tion Identifies Candidate Metrics That Influence the Human Electroencephalogram." Proceedings of the National Academy of Sciences 107, no. 19 (2010): 8695-8700.

[9] Dumermuth, G., Molinary, L., (1987), Spectral Analysis of EEG Background Activity, In Handbook Methods of Analysis of Brain Electrical and Magnetic Signals, A. S. Gevins (ed.) (Amsterdam: Elsevier).

[10] Sommer BJ, Barycki JJ, Simpson MA. Characterization of human UDP-glucose dehydroge-nase. J Biol Chem 2004;279:23590.

[11] Marcel, S., Milln, J. del R., (2007), Person authentication using brainwaves (EEG) and maximum a posteriori model adaptation. Pattern Analysis and Machine Intelligence, IEEE Transactions on 29, 743 - 752.

[12] Dougherty MK, Morrison DK. Unlocking the code of 14-3-3. J Cell Sci 2004;117:187584.

[13] Hirsch L. J., Richard P., Brenner R.P., (2010), Atlas of EEG in Critical Care, WileyBlackwell..

[14] Percival, D. B., Walden, A. T., (1993), Spectral Analysis for Physical Applications Multita-per and Conventional Univariate Techniques, Cambridge, England: Cambridge Univer-sity Press

[15] Henry Small," Co-citation in the scientific literature: A new measure of the relationship between two documents", Volume 24, Issue 4, pages 265-269, July/August 1973.

[16] Fang Li et al.," Applying Association Rule Analysis in Bibliometric Analysis-A Case Study in Data Mining", Proceedings of the 
Second Symposium International Computer Science and Computational Technology(ISCSCT '09),Huangshan, P. R. China, 26-28,Dec. 2009, pp. 431-434.

[17] Gerald Matthews, Lauren Reinerman-Jones, Julian Abich IV, Almira Kustubayeva, "Metrics for individual differences in EEG response to cognitive workload: Optimizing per-formance prediction", Personality and Individual Differences, Volume 118, 1 Novem-ber 2017, Pages 22-28

[18] Anderson S. Oliveira,Bryan R. Schlink,W. David Hairston, Peter König, and Daniel P. Ferris, "Proposing Metrics for Benchmarking Novel EEG Technologies Towards RealWorld Measurements", frontiers in Human Neuroscience

[19] Stefanidis V., Anogianakis G., Evangelou A., Poulos M., «Learning Difficulties Prediction using Multichannel Brain Evoked Potential Data», IEEE Proceedings, MCSI, pp. 268-272, 2016.

[20] Steenkamp M. M., Litz B. T., Hoge C. W., \& Marmar C. R. (2015). Psychotherapy for military-related ptsd: A review of randomized clinical trials. JAMA, 314(5), 489-500.

[21] Agatha Lenartowicz, and Sandra K. Loo, "Use of EEG to Diagnose ADHD", Curr Psychiatry Repot 2014 Nov; 16(11): 498.

[22] Jasper HH, Solomon P, Bradley C. Electroencephalographic analyses of behavior problem children. American Journal of Psychiatry. 1938;95(3):641-658.

[23] V. Stefanidis, M. Poulos, S. Papavlasopoulos (2018), Bibliometrics EEG Metrics Associations and Connections Between Learning Disabilities and the Human Brain Activity, Knowledge-Based Software Engineering, Springer Smart Innovation, Systems and Technologies book series (SIST)

[24] V. Stefanidis, M. Poulos, S. Papavlasopoulos (2018), Bibliometrics Associations between EEG Entropies and Connections between Learning Disabilities and the Human Brain Activity, International Journal of Computers, vol. 3 pp 177-181

[25] Sheerin CM, Franke LM, Aggen SH, Amstadter AB, Walker WC. Evaluating the Contribution of EEG Power Profiles to Characterize and Discriminate Posttraumatic Stress Symptom Factors in a Combat-Exposed Population. Clinical EEG Neurosciences. 2018 Nov;49(6):379-387

[26] Wang C, Ramakrishnan S, Laxminarayan S, Dovzhenok A, Cashmere JD, Germain A,
Reifman J. An attempt to identify reproducible high-density EEG markers of PTSD during sleep. Sleep. 2020 Jan 13;43

\section{Creative Commons Attribution License 4.0 (Attribution 4.0 International, CC BY 4.0)}

This article is published under the terms of the Creative Commons Attribution License 4.0 https://creativecommons.org/licenses/by/4.0/deed.en_US 